\title{
Una nueva especie de Adraneothrips (Thysanoptera, Tubulifera, Phlaeothripidae)
}

\author{
Carolina Alvarez ${ }^{1}$, Cristina Sanchez ${ }^{1} \&$ Axel P. Retana-Salazar ${ }^{2,3}$
}

1. Escuela de Biología, Universidad de Costa Rica, 2060.

2. Centro de Investigación en Contaminación Ambiental (CICA), Universidad de Costa Rica, 2060. Proyecto MISA, San José, Costa Rica. (apretana@cariari.ucr.ac.cr)

3. Centro de Investigación en Estructuras Microscópicas (CIEMIC), Ciudad de la Investigación, Universidad de Costa Rica, 2060, San José, Costa Rica.

\begin{abstract}
A new species of Adraneothrips (Thysanoptera, Tubulifera, Phlaeothripidae). A new species of Adraneothrips Hood, 1925, soil Thysanoptera, is described from Costa Rica. A key for the recognition of the species of Central America is provided.
\end{abstract}

KEYWORDS. Adraneothrips, soil thrips, Neotropics, taxonomy.

RESUMEN. En este trabajo se describe una nueva especie de thrips de suelo para Adraneothrips Hood, 1925 de Costa Rica. Se provee una clave de identificación para las especies de América Central.

PALABRAS-CLAVE. Adraneothrips, thrips de suelo, Neotrópico, taxonomía.

Phlaeothripidae es una de las familias más diversas dentro del orden Thysanoptera, con más de 3.000 especies descritas. Uno de los mayores problemas del grupo es la enorme variación morfológica que presenta, sin embargo es posible definir caracteres de peso para la determinación de las jerarquías taxonómicas (BHAтTI, 1994). La subfamilia Phlaeothripinae, tradicionalmente reconocida por el desarrollo de los estiletes, es la más diversa, dentro de ella los grupos asociados a suelos y microflora edáfica son los más raros (Mound \& MARulLo, 1996). Uno de los problemas que enfrenta el taxónomo de este grupo es el prejuicio derivado del 5\% de especies plaga que hay en el orden de que todas las especies de thrips presentan poblaciones altas.

$\mathrm{Al}$ igual que casi todo grupo la mayor parte de sus especies son raras ya que las especies de las que se recolecta entre 1 y 10 individuos son más del $80 \%$ y con números mayores a 40 o 50 individuos apenas sobrepasan el 15\% (GASTON, 1994; GUTMAN, 1999). Los muestreos en suelo suelen ser complicados tanto por la preservación de la muestra como por su extracción y en particular su clasificación a posteriori. Por esta razón y por lo poco estudiado de este hábitat en las regiones neotropicales es que es sumamente sencillo hallar nuevas especies asociadas a este medio, particularmente de microartrópodos que además presentan la dificultad de su clasificación y preservación, ya que además del trabajo de campo, exigen un gran trabajo de laboratorio.

Uno de los grupos más diversos en el suelos del neotrópico es el género Adraneothrips Hood, 1925, con cerca de 50 especies descritas, muchas de ellas a partir de ejemplares únicos que han sido recolectados solo una vez (Mound \& Marullo, 1996).

En este trabajo se presenta la descripción de una nueva especie para el género Adraneothrips del litoral atlántico costarricense, en las cercanías de bosques perennifolios al nivel del mar.

\section{MATERIALY MÉTODOS}

Se tomaron muestras de la capa humídica del suelo en bolsas plásticas para evitar la desecación. Los especimenes se separaron utilizando embudos de berlesse por periodos de hasta cuatro horas para muestras de 250 gramos. Luego se procedió a la separación de los diferentes grupos con particular énfasis en los microartrópodos, de entre los cuales se determinó la presencia de una nueva especie para la ciencia. El montaje se efectuó en Bálsamo de Canadá después de someter los especimenes a una batería de deshidratación por alcoholes. Se obtuvo una muestra de 562 microartrópodos de suelo, la mayor parte de ellos ácaros del grupo de los Oribatida. Se recolectaron siete individuos del género Adraneothrips, de los cuales tres fueron identificados como Adraneothrips gandoca sp. nov. Las fotografías se tomaron con equipo óptico binocular y con el programa Image ProExpress, para procesamiento de imágenes científicas. Los especímenes se depositaron en las siguientes colecciones institucionales: Centro de Investigación en Estructuras Microscópicas (CIEMic), Universidad de Costa Rica, Museo de Insectos de la Universidad de Costa Rica (MIUCR), Museo de Senckenberg, Frankfurt, Alemania (SMF).

\section{Adraneothrips gandoca sp. nov. (Figs. 1-8)}

Etimología. Esta especie recibe su nombre de la localidad del caribe costarricense en la que fue recolectada por vez primera.

Material tipo. Holotipo. + , COSTA RICA, Limón: GandocaManzanillo, macróptera, IX-X/2005, C. Álvarez y C. Sánchez. Cols. (CIEMic). Paratipos. Limón: Gandoca-Manzanillo,, , ơ macróptera, ix-x/2005, C. Álvarez y C Sánchez. Cols. (†, SMF; ${ }^{7}$, MIUCR). 

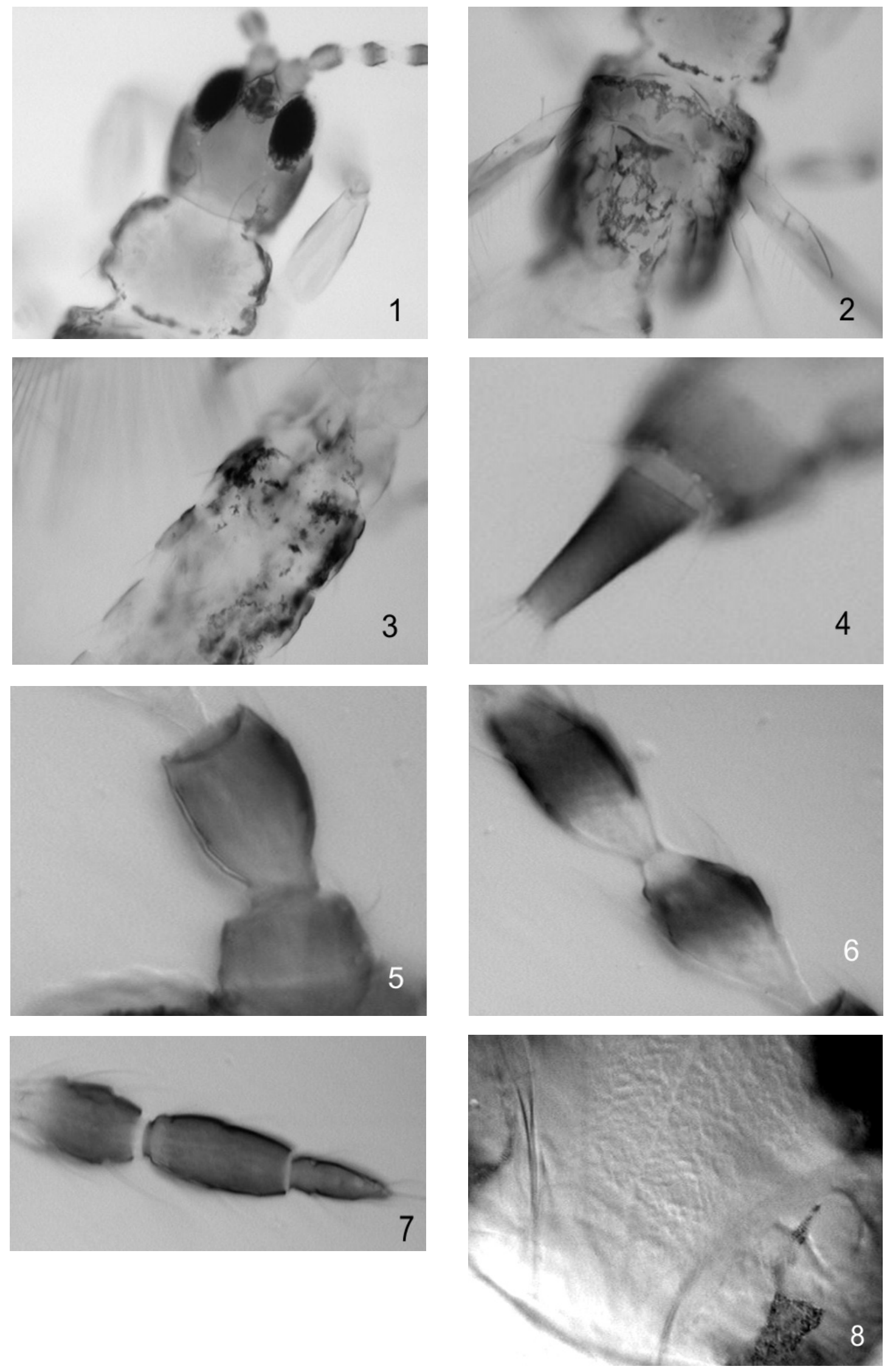

Figs. 1-8. Adraneothrips gandoca sp. nov. 1, cabeza y pronoto; 2, pterotórax; 3, segmentos abdominales; 4, segmento X del abdomen; 5-7, antenómeros: 5, I-II; 6, III-IV; 7, VI-VIII; 8, estiletes maxilares. 
Descripción. Longitud total del cuerpo $1.300 \mu \mathrm{m}$. Color: cabeza y pronoto (Fig. 1) amarillo ámbar. Meso- y metatórax (Fig. 2) de color café con rojo subtegumentario. Abdomen amarillo (Fig. 3) excepto el segmento X (Fig. 4). Alas traslúcidas con tres cilios accesorios en el borde posterior. Patas todas amarillas. Antenómeros III-IV con cuatro (4) sensores en cada uno. Antenómeros I-II café (Fig. 5), III-VI bicolores con el tercio basal amarillo y los dos tercios distales de color café (Fig. 6), VII-VIII café oscuro (Fig. 7). Ancho de la cabeza $135 \mu \mathrm{m}$ (parte más ancha detrás de los ojos), separación entre los estiletes $40 \mu \mathrm{m}$ (Fig. 8). Pelta más larga que ancha, pero no tiene forma de campana ni sensilas campaniformes. Presenta los cinco pares de setas bien desarrolladas del pronoto.

Comentario. Esta especie pertenece al grupo Adraenothrips alternatus Hood, 1935, que se caracteriza por un patrón de color donde los lados de la cabeza y el pronoto presentan bordes fuertemente pigmentados mientras que la sección central es clara. Adraneothrips gandoca sp. nov. presenta la particularidad de que el color amarillo es en realidad ámbar gracias a la presencia de pigmento rojo subtegumentario, además cuenta con la presencia de cuatro sensores en los segmentos III-IV de la antena. La distancia entre los estiletes maxilares es aproximadamente 0,2 del ancho de la cabeza.

Clave de identificación para las especies de Adraneothrips en Centro América

1. Especies con el cuerpo de color café ............................ 2 Especies con patrón bicolor ....................................... 5

2. Tibia y tarso amarillos en contraste con el fémur ...... 3

Tibia y tarso café o no más claros que le fémur ........ 4

3. Setas anteromarginales (am) bien desarrolladas con los ápices expandidos A. tibialis Hood, 1914

Setas anteromarginales $(\mathrm{am})$ reducidas con los ápices agudos ......... A. acutulus Mound \& Marullo, 1996

4. Ojos proyectados en la cara ventral de la cabeza; estiletes cortos y nunca retraídos detrás de los ojos; antenómeros IV-V café en la base, seta B1-B2 del segmento IX con el ápice agudo

................................. A. microsetis Hood, 1942

Ojos nunca proyectados en la cara ventral de la cabeza; estiletes largos y fuertemente retraídos detrás de los ojos; antenómeros IV-VI café en su totalidad, setas B1-B2 del segmento IX con el ápice expandido A. obliquus Hood, 1950

5. Antenómero III con 4 conos sensoriales; cabeza y pronoto claros con bordes pigmentados con café oscuro en las secciones laterales; mesonoto y metanoto café con rojo subtegumentario; abdomen amarillo, sin marcas laterales en café

A. gandoca sp.nov.

Antenómero III con 3 conos sensoriales; cabeza café, pronoto de un solo color amarillo o café; mesonoto y metanoto variable en coloración pero nunca con rojo subtegumentario; abdomen variable en coloración, pero con márgenes café ...................... 6

6. Ojos proyectados ventralmente ................................ 7

Ojos no proyectados ventralmente (a veces adelgazados en la parte posterior) ... 9

7. Pronoto amarillo, antenómero III con 3 sensores ..... A. brasiliensis Hood, 1950

Pronoto café, antenómero III con 2 sensores ........... 8

8. Patas amarillas ..................... A. diligens Hood, 1935

Patas café ........... A alajuela Mound \&Marullo, 1996

9. Cabeza aproximadamente 1,3 veces más larga que ancha; antenómero III amarillo, IV-VI café; estiletes maxilares muy cercanos entre sí; pronoto amarillo A. albicollis Hood, 1935

Cabeza nunca mucho más larga que ancha; antenómeros variables en coloración pero nunca con la combinación anterior; estiletes maxilares ampliamente separados por un $30 \%$ del ancho de la cabeza; pronoto variable en color .. 10

10.Pelta sin sensilas campaniformes y truncada basalmente delante del tergito II; el pronoto es variable en coloración, desde café claro hasta amarillo

A. alternatus Hood, 1925

Pelta con sensilas campaniformes; pronoto café ..... 11

11. Setas anteroangulares del pronoto de 30 micrómetros o más, tan largas como la parte basal más angosta de la pelta A. fuscicollis Hood, 1925

Setas anteroangulares del pronoto de 25 micrómetros o menos, cerca de la mitad de la sección basal más angosta de la pelta. A. biadenes Mound \& Marullo, 1996

Agradecimientos. Al señor Jeans Espinoza por su colaboración en Sistemas de Cómputo, al señor Jerson GaritaCambronero por su ayuda en la elaboración del texto gráfico; al señor Fernando Ortiz Morales del Instituto de Investigaciones en Salud (INISA) de la Universidad de Costa Rica. Al Proyecto MISA (Microartrópodos como Indicadores de Salud Ambiental, CICA) por el apoyo logístico de este trabajo y al proyecto Descrispcíon y ultrestructura de los Thrips (Insecta: Thysanoptera) de Mesoamérica 810-A6-239 por su colaboración.

\section{REFERENCIAS BIBLIOGRÁFICAS}

Bнатті, J. S. 1994. Phylogenetic relationships among Thysanoptera (Insecta) with particular reference to the families of the Order Tubulifera. Journal of Pure and Applied Zoology 4:93-130.

Gaston, K. 1994. Rarity. Population and Community Biology Series $\mathrm{N}^{\circ}$ 13. London, Chapman and Hall. 205p.

Gutman, B. S. 1999. The Structure of Biological Communities. New York, McGraw-Hill. 578p.

Mound, L. A. \& Marullo, R. 1996. The thrips of Central and South America: an introduction (Insecta: Thysanoptera). Florida, Associated Publishers. 487p. (Memoirs on Entomology International 6).

Recebido em setembro de 2006. Aceito em dezembro de 2006. ISSN 0073-4721

Artigo disponível em: www.scielo.br/isz

Iheringia, Sér. Zool., Porto Alegre, 97(3):243-245, 30 de setembro de 2007 